\title{
End Stage Liver Disease
}

National Cancer Institute

\section{Source}

National Cancer Institute. End Stage Liver Disease. NCI Thesaurus. Code C84428.

A disorder characterized by irreversible damage to the liver tissue, leading to complete

liver failure. Causes include cirrhosis, viral hepatitis, metastases to the liver, genetic disorders, toxins, and drugs. 